\title{
Convergent Expansions for Tunneling
}

\author{
J. M. Combes`, P. Duclos`, and R. Seiler ${ }^{\star \star}$
}

Centre de Physique Théorique, CNRS - Luminy, Case 907, F-13288 Marseille Cedex 9, France

\begin{abstract}
A new method to compute effects of tunneling in one-dimensional multiple well is developed. A tunneling parameter built with physical quantities is introduced to measure the splitting between eigenvalues due to tunneling. These splittings are given by convergent series in term of this tunneling parameter for a wide class of double well.
\end{abstract}

\section{Introduction}

This is our second article in a series about the classical limit of one dimensional Schrödinger operators with multiple well potentials. The main subject of this article is tunneling. We shall use some of the results proved in the first paper of this series, referred to as [CDS 1]; it concerns the harmonic approximation, the exponential decay of eigenfunctions and asymptotic series for eigenvalues in the classical limit.

The third paper will treat tunneling in the case of shape resonances.

To describe more precisely what we mean by tunneling let us consider the Schrödinger operator

$$
H(k)=-k^{4} \Delta+V
$$

on the interval $\Omega$ of the real line $\mathbb{R}$, where $V$ is a potential function with an at most doubly degenerate absolute minimum $v_{0}$. Typical graphs of $V$ for $v_{0}=0$ are shown in Fig. 1.

In the following we state the precise assumptions on $V$ :

Assumptions on $V$. Let $\Omega$ be an arbitrary open interval and let $V$ satisfy

i) $V \in L_{\mathrm{loc}}^{1}(\Omega)$.

ii) $V$ has an absolute minimum $v_{0}$ which can be at most degenerate of order 2 .

* PHYMAT, Département de Mathématiques, Université de Toulon et du Var, and Centre de Physique Théorique, CNRS, F-13288 Marseille, France

$\star \star$ Institut für Theoretische Physik, Freie Universität, D-1000 Berlin 

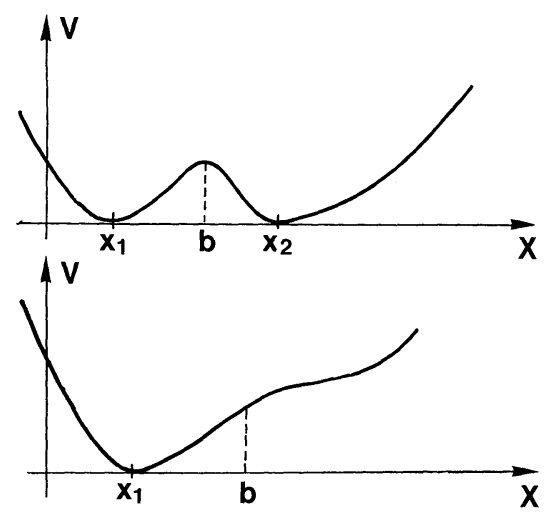

Fig. 1

We split $\Omega$ in two intervals $\Omega_{1}$ and $\Omega_{2}$

$$
\bar{\Omega}=\bar{\Omega}_{1} \cup \bar{\Omega}_{2}, \quad \Omega_{1}=(a, b), \quad \Omega_{2}=(b, c), \quad a<b<c,
$$

such that $V$ has a nondegenerate minimum $v_{0}$ at $x_{1}$ inside $\Omega_{1}$. In case of degeneracy the second point where $V$ takes its minimal value is inside $\Omega_{2}$ and called $x_{2}$.

iii) $V$ admits asymptotic expansions up to order $N \geqq 2$ at every $x_{i}$

$$
V(x)=v_{0}+\sum_{n=2}^{N} v_{n, i}\left(x-x_{i}\right)^{n}+O\left(\left|x-x_{i}\right|^{N+1}\right), \quad v_{2, i}>0 .
$$

The definition of $H(k)$ under the above conditions on $V$ is given in Chap. II. Then we introduce the operator $H^{D}(k)$ with the same differential symbol as $H(k)$ but an additional Dirichlet boundary condition at the points $b$ defined in (2). This boundary condition decouples the two intervals $\Omega_{1}$ and $\Omega_{2}$ by what is physically interpreted as an infinitely high and narrow wall. Particles are thereby confined to their respective wells. By definition $H^{D}(k)$ is not affected by tunneling from one well to the other, because

$$
H^{D}(k)=H_{1}^{D}(k) \oplus H_{2}^{D}(k),
$$

and $H^{D}(k)$ is at the same time very close to $H(k)$. Therefore we shall take $H^{D}(k)$ as a point of reference in our discussion of tunneling. Comparing eigenvalues and eigenfunctions of $H(k)$ and $H^{D}(k)$ we shall attribute any change to tunneling. Mathematically tunneling is introduced by a change of boundary conditions.

In this article we present results about discrete eigenvalues of $H(k)$ in the terms of $H^{D}(k)$ in the limit $k \downarrow 0$. We shall prove that every spectral valued function of $k$, $E(k) \in \sigma(H(k))$ is given by a convergent power series in the tunneling parameter $t(k)$ (Theorems III.1.2, III.2.2, and III.2.4). $t(k)$ is defined in Definitions II.6 and II.8 and proved to be exponentially small in $k^{-1}: t(k)=o\left(\exp -\right.$ const $\left.k^{-2}\right)$.

The method used in the analysis of the spectrum of $H(k)$ in the terms of $H^{D}(k)$ is the following. The resolvents of $H(k)$ and $H^{D}(k)$ are related by Krein's formula [Kr, AG, Ka, p. 188]

$$
(H+1)^{-1}=\left(H^{D}+1\right)^{-1}+\Pi
$$


where $\Pi$ is a rank one operator and incorporates the effects of tunneling. The spectrum of $H$ is therefore intimately related to the zeros of the WeinsteinAronszajn determinant $\omega(z)$ associated with $\left(H^{D}+1\right)^{-1}$ and $\Pi$ (see [Ka, p. 244] and references therein), which are the poles of the resolvent of $(H+1)^{-1}(\mathrm{II} .15-17)$. It is shown that $\omega(z)$ has a particularly nice structure, so that the zero set can be analysed by means of Lagrange's inversion formula $[D]$. It turns out that the proofs and results are sensitive to whether the spectrum valued function $E^{D}(k)$ of $H^{D}(k)$ we start from is degenerate or nondegenerate in the limit $k \downarrow 0$. This concept is introduced in Definition II.4 and means roughly the following: $E^{D}(k)$ is called degenerate in the limit if there is a second spectral valued function $\tilde{E}^{D}(k)$ such that (for $v_{0}=0$ )

$$
\lim _{k \rightarrow 0} k^{-2} E^{D}(k)=\lim _{k \rightarrow 0} k^{-2} \tilde{E}^{D}(k) .
$$

Otherwise $E^{D}(k)$ is nondegenerate in the limit. The fact that the above limit always exists follows from our results about the harmonic approximation [CDS 1]. In the case of the symmetric double well every spectral valued function is degenerate and therefore degenerate in the limit. The analysis of the zero set of $\omega(z)$ is given in Chap. III, Sects. 1 and 2 for the cases of spectral valued functions $E^{D}(k)$ nondegenerate in the limit and degenerate in the limit, respectively.

Some words about multi-instanton expansions and possible extensions of this work are given in the concluding remarks of Chap. IV.

The main motivation for our analysis comes from our work on the BornOppenheimer approximation [CDS 0]. In this case the parameter $k^{4}$ is the ratio of the electronic to the nuclear mass. Another motivation was our aim to give a rigorous and systematic version of Polyakov's method of instantons to compute the energy splitting of the two lowest eigenvalues for the symmetric double-well (see $[\mathrm{P}]$ and references therein). A particularly nice application of results about the classical limit is Witten's derivation of the Morse inequalities [W]. For that one needs, however, a generalization of our results to many dimensions. This has been done by Simon using geometric methods [S 1].

There is a large literature on this model which has been used in many different branches of physics. In particular Harrell [H 1$]$ has given a rigorous version of the WKB approach and computed all the contributions to the energy eigenvalues up to first order in the tunneling parameter. Jona-Lasinio et al. [JMS] used stochastic methods to prove localization of eigenfunctions and asymptotic series for discrete eigenvalues. Zinn-Justin developed the method of instantons to get various formulae for eigenvalues and tested many of them numerically [Z]. In $[\mathrm{R}-\mathrm{R}]$, complex instantons are used to compute the first order in the tunneling parameter of the splitting between the two first eigenvalues of the anharmonic oscillator. Also, a systematic analysis of double well Hamiltonians in a more abstract setting can be found in a recent series of papers by Davies (see [Da]).

\section{Objects of Interest and some of their Properties}

As we have said in the Introduction we want to link eigenvalues of $H(k)$ to the ones of $H^{D}(k) . H(k)$ is the positive self-adjoint operator with symbol $-k^{4} \Delta+V$ defined 
in $L^{2}(\Omega), \Omega$ open interval, and Dirichlet boundary condition on $\partial \Omega$. The assumption on $V$ are made in Chap. I. By Dirichlet boundary condition on $\partial \Omega$ in the case of singular behavior of $V$ at the boundary we mean the Friedrichs extension of the operator with the same symbol and domain $C_{0}^{\infty}(\Omega)$ (see $[\mathrm{Ka}$, p. 343]).

Without loss of generality we shall take in the following

$$
v_{0}=0, \quad x_{1}=0<b<x_{2} .
$$

Thus, $V$ will be positive.

Remark 1 . To clarify what we mean by an absolute minimum $v_{0}$ of a $L_{\text {loc }}^{1}$ function we describe it below:

$$
\begin{gathered}
\operatorname{ess} \inf \{V(x), x \in \Omega\}=v_{0} \\
\forall \Omega^{\prime} \subset \Omega, \Omega^{\prime} \text { closed interval, } x_{i} \notin \Omega^{\prime}, \text { then } \\
\text { ess inf }\left\{V(x), x \in \Omega^{\prime}\right\}>v_{0} .
\end{gathered}
$$

We stress that if we introduce

$$
\begin{gathered}
V_{ \pm}= \begin{cases}\liminf _{x \rightarrow \pm \infty} V(x) & \text { if } \pm \infty \in \bar{\Omega} \\
+\infty & \text { if not, }\end{cases} \\
V_{\infty}=\min \left(V_{+}, V_{-}\right),
\end{gathered}
$$

then (2) implies

$$
V_{\infty}>v_{0}
$$

Then $H^{D}(k)$ is obtained from $H(k)$ by adding an extra Dirichlet boundary condition at the point $b$ mentioned in the assumption on $V$.

The microscopic operators $h(k)$ and $h^{D}(k)$ are technically convenient. They are defined by

$$
h(k)=k^{-2} D(k) H(k) D^{-1}(k) \text { and similarly for } h^{D}(k),
$$

where $D(k)$ denotes the unitary dilation operator from $L^{2}(\Omega)$ to $L^{2}(\Omega(k)), \Omega(k)$ $=k^{-1} \Omega$, and

$$
(D(k) u)(x)=k^{1 / 2} u(k x),
$$

Their symbols will be denoted by $-\Delta+W(k, x)$. This procedure introduces an artificial distinction between the two wells when the minimum is degenerate. In this case a second microscopic operator can be defined by setting the origin of dilation to the second position $x_{2}$ of the minimum. However, the so-defined microscopic operators are unitarily equivalent. Hence we can restrict ourself to the analysis of $h(k)$ defined by (6).

For $h^{D}(k)$ one has a direct sum decomposition

$$
h(k)=h_{1}^{D}(k) \oplus h_{2}^{D}(k) \text {. }
$$


Formulating the first result on these operators we have to introduce the two following harmonic oscillator operators $h_{i}^{(2) 1}$ with symbol $-\Delta+v_{2, i} x^{2}, i=1,2$. In case $V$ does not take its minimal value inside $\Omega_{2}$, we formally put $h_{2}^{(2)}=\infty$; by this we mean vanishing of its resolvent. Let $r(k), r^{D}(k), r_{i}^{D}(k), r_{i}^{(2)}, i=1,2$, denote the resolvent at the point $z=-1$ of $h(k), h^{D}(k), h_{i}^{D}(k), h_{i}^{(2)}$.

Then

Proposition 2. i) $r(k), r^{D}(k)$ "converge as $k \downarrow 0$ " to $r_{1}^{(2)}$ in norm when the minimum of $V$ is nondegenerate and strongly otherwise.

ii) If $T\left(k^{-1} x_{2}\right)$ denotes the translation operator

$$
\left(T\left(k^{-1} x_{2}\right) u\right)(x)=u\left(x-k^{-1} x_{2}\right),
$$

then $r_{1}^{D}(k)$ and $T\left(k^{-1} x_{2}\right) r_{2}^{D}(k) T\left(-k^{-1} x_{2}\right)$ "converge as $k \downarrow 0$ " in norm to $r_{1}^{(2)}$ and $r_{2}^{(2)}$, respectively.

$$
\lim _{k \rightarrow 0}\left\|r(k)-r^{D}(k)\right\|=0 .
$$

The signification of the inverted comma on the expression "converges as $k \downarrow 0$ " is given in the beginning of the proof.

Proof. $r(k)$ and $r^{D}(k)$ do not live in the same Hilbert space as $r_{1}^{(2)}$, when $\Omega \neq \mathbb{R}$; this makes improper the expression "converge as $k \downarrow 0$." To make it proper we complete the operator in all $L^{2}(\mathbb{R})$ by an arbitrary one which norm converges to zero as $k \downarrow 0$. Further details on this procedure may be found in [CDS 1, Chap. III, Sect. 1]. A similar remark works for $r_{1}^{D}(k)$ and $r_{2}^{D}(k)$.

iii) is a direct consequence of Propositions 6 and 7 of Chap. I in [CDS 1]. If $r(k)(x, y)$ denotes the integral kernel of $r(k)$ one has

$$
\left\|r(k)-r^{D}(k)\right\| \leqq r(k)\left(k^{-1} b, k^{-1} b\right)=O\left(k^{1 / 2}\right) .
$$

This is based on Krein's formula and an asymptotic estimate on the diagonal of the kernel of $r(k)$ at $x=k^{-1} b$. Because of iii) it is sufficient to show i) for $r(k)$. When the minimum of $V$ is nondegenerate one may paraphrase the proof of Theorem 2.2, Chap. III in [CDS 1]. The method uses a pointwise estimate via Feynman Kac formula on the kernel of $r(k)-r_{1}^{(2)}$. Otherwise when the minimum of $V$ is degenerate the strong convergence needs only to be verified on $C_{0}^{\infty}(\mathbb{R})$ because

$$
\left\|r(k)-r_{1}^{(2)}\right\| \leqq 2 \text {. }
$$

Then the same method applies with slight modifications. The proof of ii) is similar to the one of i).

Eigenvalues of $H(k), H^{D}(k), h(k), h^{D}(k), r(k), r^{D}(k)$ will be denoted generically by $E(k), E^{D}(k), e(k), e^{D}(k), f(k), f^{D}(k)$. The ones of $h_{i}^{(2)}, r_{i}^{(2)}, i=1,2$ will be denoted by $e^{(2)}$ and $f^{(2)}$. A crucial role will be played by the sets

$$
\mathscr{L}=\sigma\left(h_{1}^{(2)}\right) \cup \sigma\left(h_{2}^{(2)}\right) ; \quad(\mathscr{L}+1)^{-1}=\sigma\left(r_{1}^{(2)}\right) \cup \sigma\left(r_{2}^{(2)}\right) .
$$

One important consequence of Proposition 2 is:

1 The subscript (2) on the $h_{i}^{(2)}$ 's is there to recall that they are the second order approximation to $h(k)$ with respect to the expansion in $k$ 
Proposition 3. $\mathscr{L}$ is exactly the limit point set of all the eigenvalues of either $h(k)$ or $h^{D}(k)$.

Eigenvalues in $\mathscr{L}$ are stable in the sense of Kato [Ka, pp. 437]. In fact we use a slight generalization of his concept.

The proof can be found in [CDS 1, Chap. III, Theorem 6.8].

We shall use Proposition 2 in the following equivalent form: The set of limits: $\lim _{k \succ 0} k^{-2} E(k)$, where $E(k)$ is a spectrum valued function of either $H(k)$ or $H^{D}(k)$ is $k \searrow 0$ exactly equal to $\mathscr{L}=\sigma\left(h_{1}^{(2)}\right) \cup \sigma\left(h_{2}^{(2)}\right)$. Notice that the set of limits for $H(k)$ and $H^{D}(k)$ are equal. The spectrum valued function will be abbreviated as the s.v. function.

We introduce the following terminology:

Definition 4. An element of $\mathscr{L}=\sigma\left(h_{1}^{(2)}\right) \cup \sigma\left(h_{2}^{(2)}\right)$ is called degenerate if it belongs to both spectra $\sigma\left(h_{1}^{(2)}\right)$ and $\sigma\left(h_{2}^{(2)}\right)$, otherwise it is called nondegenerate. A spectrum valued function $E(k)$ of $H^{D}(k)$ is called degenerate in the limit if $\lim _{k \rightarrow 0} k^{-2} E(k)$ is a degenerate element of $\mathscr{L}$, otherwise $E(k)$ is called nondegenerate in the limit.

Remark 5. i) The degeneracy of elements of $\mathscr{L}$ is at most 2.

ii) The notion of degeneracy in the limit can be analogously introduced for s.v. functions of $H(k)$. Though the spectrum of $H(k)$ is never degenerate, it may have s.v. functions degenerate in the limit.

From now on we shall not write the explicit dependence in $k$ in the objects to be considered when we feel it is not necessary. According to our general strategy we shall use Krein's formula which relates the resolvents of the microscopic operators $h$ and $h^{D}$

$$
r=r^{D}+\pi
$$

where $\pi$ is the rank one operator whose integral kernel is

$$
\pi(x, y)=\tau(x) \tau(y) ; \quad \tau(x)=r\left(k^{-1} b, k^{-1} b\right)^{-1 / 2} r\left(k^{-1} b, x\right) .
$$

We shall use in the following the W-A formula (see [Ka, p. 244]) for which we need the W-A determinant associated with $r^{D}$ and $\pi$ :

$$
\begin{aligned}
\omega(z) & =\omega\left(z ; r^{D}, \pi\right)=\operatorname{det}\left(1+\pi\left(r^{D}-z\right)^{-1}\right) \\
& =1+\operatorname{tr} \pi\left(r^{D}-z\right)^{-1} \\
& =1+\left(\left(r^{D}-z\right)^{-1} \tau, \tau\right)
\end{aligned}
$$

Let us recall briefly some consequences of these formulae

$$
\begin{gathered}
z \in \sigma\left(r^{D}\right) \text { with multiplicity one, then } z \in \varrho(r), \\
z \in \sigma\left(r^{D}\right) \text { with multiplicity two, then } z \in \sigma(r), \\
z \in \varrho\left(r^{D}\right) \text {, then } z \in \sigma(r) \Leftrightarrow \omega(z)=0 .
\end{gathered}
$$

For the case at hand the above results can be deduced by an analysis of the following formula

$$
(r-z)^{-1}=\left(r^{D}-z\right)^{-1}+\frac{\left(r^{D}-z\right)^{-1} \pi\left(r^{D}-z\right)^{-1}}{\omega(z)} .
$$


Proposition 3 shows that the eigenvalues of $h$ and $h^{D}$ are related through their limit points in $\mathscr{L}$. In Chap. III we shall elaborate on this relation. This will be done by looking at the zeros of $\omega$. For that purpose we have to analyse more precisely the structure of $\omega$. From now on this analysis relies on the type of degeneracy in the limit of the s.v. functions (Definition 4).

Structure of W-A Determinant in the Nondegenerate Situation. Let $f^{D} \in \sigma\left(r^{D}\right)$ such that $\lim f^{D}(k)=f^{(2)} \in(\mathscr{L}+1)^{-1}$, where $f^{(2)}$ is nondegenerate. Let $\varphi^{D}, P^{D}$ denote the corresponding normalized eigenvector and eigenprojection. Then

$$
\omega(z)=1+\frac{\operatorname{tr} \pi P^{D}}{f^{D}-z}+\operatorname{tr} \pi\left(\widehat{r^{D}-z}\right)^{-1}
$$

where $\left(\widehat{r^{D}-z}\right)^{-1}$ denotes the corresponding reduced resolvent of $r^{D}$. We introduce two new objects:

Definition 6. We shall call $\operatorname{tr} \pi P^{D}$ the tunneling parameter associated to $f^{D}$ and denote it by

$$
t=\operatorname{tr} \pi P^{D}=\left|\left(\varphi^{D}, \tau\right)\right|^{2} .
$$

We introduce also the function $\sigma$ defined by

$$
\begin{aligned}
\sigma^{-1}(z) & =1+\operatorname{tr} \pi\left(\widehat{r^{D}-z}\right)^{-1} \\
& =1+\left(\left(\overline{r^{D}-z}\right)^{-1} \tau, \tau\right) .
\end{aligned}
$$

Thus $\omega$ takes the form

$$
\omega(z)=\frac{t}{f^{D}-z}+\sigma^{-1}(z)
$$

Remark 7. $\sigma^{-1}$ is precisely the $\mathrm{W}-\mathrm{A}$ determinant associated with $\hat{r}^{D}$ and $\pi$.

Structure of the W-A Determinant in the Degenerate Situation. Let now $f_{i}^{D}, i=1,2$, be two eigenvalues of $r_{i}^{D}$ such that $\lim _{k \rightarrow 0} f_{i}^{D}=f^{(2)} \in(\mathscr{L}+1)^{-1}$. Let $\varphi_{i}^{D}, P_{i}^{D}$, be the corresponding normalized eigenvectors and eigenprojections. Then

$$
\omega(z)=1+\frac{\operatorname{tr} \pi P_{1}^{D}}{f_{1}^{D}-z}+\frac{\operatorname{tr} \pi P_{2}^{D}}{f_{2}^{D}-z}+\operatorname{tr} \pi\left(\widehat{r^{D}-z}\right)^{-1},
$$

where $\left(\widehat{r^{D}-z}\right)^{-1}$ denotes, as before, the reduced resolvent of $r^{D}$.

Definition 8 . We shall call $\operatorname{tr} \pi P_{i}^{D}, i=1,2$ the tunneling parameters associated to $f_{i}^{D}$ and denote them by

$$
t_{i}=\operatorname{tr} \pi P_{i}^{D}=\left|\left(\varphi_{i}^{D}, \tau\right)\right|^{2} .
$$

Then we shall call $t=t_{1}+t_{2}$ the total tunneling parameter associated to the group $f_{i}^{D}, i=1,2$. Finally we introduce the function $\sigma$ defined by

$$
\sigma^{-1}(z)=1+\operatorname{tr} \pi\left(\widehat{r^{D}-z}\right)^{-1}=1+\left(\left(\widehat{r^{D}-z}\right)^{-1} \tau, \tau\right) .
$$


Thus $\omega$ takes the form

$$
\omega(z)=\frac{t_{1}}{f_{1}^{D}-z}+\frac{t_{2}}{f_{2}^{D}-z}+\sigma^{-1}(z) .
$$

Instead of using this form we prefer to do the following: in $\operatorname{Ran} P_{1}^{D} \oplus \operatorname{Ran} P_{2}^{D}$ we may look for an orthonormal basis $\{\psi, \theta\}$ such that

$$
\theta \perp \tau \text {. }
$$

We obtain

$$
\left(\begin{array}{l}
\psi \\
\theta
\end{array}\right)=\left(\begin{array}{cc}
\alpha_{1} & \alpha_{2} \\
-\alpha_{2} & \alpha_{1}
\end{array}\right)\left(\begin{array}{l}
\varphi_{1}^{D} \\
\varphi_{2}^{D}
\end{array}\right)
$$

with

$$
\alpha_{i}^{2}=\frac{t_{i}}{t_{1}+t_{2}}=\frac{t_{i}}{t} \quad \text { and } \quad t=|(\psi, \tau)|^{2}
$$

Remark 9. $\psi$ and $\theta$ in general are no longer eigenvectors of $h^{D}$. To set the final form of $\omega$ we introduce

$$
g=\alpha_{2}^{2} f_{1}^{D}+\alpha_{1}^{2} f_{2}^{D}=\frac{t_{2} f_{1}^{D}+t_{1} f_{2}^{D}}{t_{1}+t_{2}}
$$

then

$$
\omega(z)=\frac{t(z-g)}{\left(z-f_{1}^{D}\right)\left(z-f_{2}^{D}\right)}+\sigma^{-1}(z)
$$

Some Properties of the Tunneling Parameter and the Function $\sigma$

Proposition 10. The tunneling parameters $t, t_{1}, t_{2}$ and the function $\sigma^{-1}$ defined in Definitions 6 and 8 are analytic in

$$
\left\{k \in \mathbb{C},|\operatorname{Arg} k|<\frac{\pi}{8}\right\} \text {. }
$$

Proof. By standard arguments one can prove that $r(k), r_{i}^{D}(k), i=1,2$, are analytic families of type $B$ (see [Ka, p. 395]) at least in the sector

$$
\left\{k \in \mathbb{C},|\operatorname{Arg} k|<\frac{\pi}{8}\right\} \text {. }
$$

Then one deduces easily the analyticity for total eigenprojections and $\pi(k)$.

We now give estimates on the tunneling parameters.

Proposition 11. One has in the nondegenerate situation

$$
t(k)=o\left(\exp -2 \beta k^{-2} \int_{0}^{b} V(s)^{1 / 2} d s\right), \quad \beta<1,
$$


and in the degenerate situation

$$
t_{i}(k)=o\left(\exp -2 \beta k^{-2}\left|\int_{x_{i}}^{b} V(s)^{1 / 2} d s\right|\right), \quad i=1,2, \quad \beta<1 .
$$

Proof. These estimates are easily obtained with the complex boost method (see [CDS 1, Chap. III, Sect. 3]). The method here is pushed further to obtain exponential decay on Green's functions. Here we give the proof only for $t(k)$.

For any $\alpha$ with $|\operatorname{Im} \alpha|<1$ we defined the multiplication operators

$$
U(\alpha)=\exp i \alpha \int_{0}^{x} W(k, t)^{1 / 2} d t,
$$

called a boost. Let $r(\alpha)$ and $r^{D}(\alpha)$ be the holomorphic families defined by

$$
r(\alpha)=U(\alpha) r U(-\alpha) ; \quad r^{D}(\alpha)=U(\alpha) r^{D} U(-\alpha) .
$$

One has the uniform estimate

$$
\|r(\alpha)\| \text { and }\left\|r^{D}(\alpha)\right\| \leqq 1 \text { for }|\operatorname{Im} \alpha|<1 .
$$

If $g(x, y)$ denotes the kernel of $r$, then the kernel of $r(\alpha)$ is

$$
g(\alpha ; x, y)=\left(\operatorname{expi\alpha } \int_{y}^{x} W(k, t)^{1 / 2} d t\right) g(x, y) .
$$

Then we defined $\tau(\alpha)$ by $^{2}$

$$
\tau(\alpha ; x)=g\left(k^{-1} b, k^{-1} b\right)^{-1 / 2} g\left(\alpha ; x, k^{-1} b\right) .
$$

Then if $\varphi^{D}(\alpha)$ denotes $U(\alpha) \varphi^{D}$, one has

$$
t(k)=\left|\left(\varphi^{D}(\alpha), \tau(\bar{\alpha})\right)\right|^{2} \exp 2 \operatorname{Im} \alpha k^{-2} \int_{0}^{b} V(s)^{1 / 2} d s .
$$

From (31) we deduce in the appendix the following uniform estimate on $g(\alpha ; \cdot, y)$

$$
\|g(\alpha ; \cdot, y)\| \leqq 1 \quad y \in k^{-1} \Omega, \quad|\operatorname{Im} \alpha| \leqq 1 .
$$

If one recalls that $\varphi^{D}(\alpha)$ is bounded in $k$ (see [CDS 1, Chap. III, Sect. 4]), then the statement for $t(k)$ follows easily.

\section{Expansions in the Tunneling Operator}

In this chapter we derive convergent expansions in the tunneling parameter for the shift between eigenvalues of $H^{D}(k)$ and their corresponding ones of $H(k)$. The analysis depends on the type of degeneracy in the limit of eigenvalues we are considering.

\section{The Nondegenerate Situation}

In this section we investigate the situation of s.v. functions which are not degenerate in the limit. Let $E^{D}$ and $E$ be s.v. functions of $H^{D}(k)$ and $H$ such that

$$
\lim _{k \rightarrow 0} k^{-2} E(k)=\lim _{k \rightarrow 0} k^{-2} E^{D}(k)=e^{(2)} \in \mathscr{L} .
$$

2 Notice that $\tau(\alpha) \neq U(\alpha) \tau$. The reason is that $\tau$ is transformed like a kernel and not like a vector 
To reconstruct $E$ starting from $E^{D}$ we prefer to work with the corresponding s.v. function $f, f^{D}$ of the resolvents of the microscopic operators. We shall use the following:

Lemma 1.1. Let $f^{(2)}=\left(e^{(2)}+1\right)^{-1}$. There exists a neighborhood $\mathscr{V}$ of $f^{(2)}$ and $k_{0}>0$ such that for any $k$ in $\left(0, k_{0}\right)$, the zero sets in $\mathscr{V} \backslash f^{D}(k)$ of $\omega(z)=1+\left(\left(r^{D}-z\right)^{-1} \tau, \tau\right)$ and $\tilde{\omega}(z)=f^{D}-z-t \sigma(z)$ coincide.

Proof. One has obviously the identity

$$
\omega(z)=\left(f^{D}-z\right)^{-1} \sigma^{-1}(z) \tilde{\omega}(z)
$$

where $t$ and $\sigma(z)$ are defined in Definition I.6. The statement follows from

$\lim _{k \rightarrow 0} \sigma(k, z)=1$, uniformly in $z$ in sufficiently small neighborhoods of $f^{(2)}$.

To prove (1.3) one looks at

$$
\left|\sigma^{-1}(z)-1\right|=\left|\left(\left(\widehat{r^{D}-z}\right)^{-1} \tau, \tau\right)\right| \leqq\left\|\left(\widehat{r^{D}-z}\right)^{-1}\right\|\|\tau\|^{2}
$$

The reduced resolvent $\left(\widehat{r^{D}-z}\right)^{-1}$ is uniformly bounded for $k$ and $\left|z-f^{(2)}\right|$ sufficiently small since $f^{(2)}$ is nondegenerate by assumption. Now $\|\tau\|$ is $O\left(k^{1 / 2}\right)$. This is a simple consequence of Propositions 6 and 7 in [CDS 1 ].

The main result of this section is contained in the following

Theorem 1.2. Let $E$ be a s.v. function of $H$ which is nondegenerate in the limit, and $f=\left(k^{-2} E+1\right)^{-1}$. Let $E^{D}$ and $f^{D}$ be the corresponding objects for $H^{D}$. Then for sufficiently small $k$ one has

$$
f=f^{D}+\sum_{n=1}^{\infty} \frac{t^{n}}{n !} \sigma_{n},
$$

where

$$
\sigma_{n}=\left.\frac{d^{n-1}}{d z^{n-1}}(\sigma(z))^{n}\right|_{z=f^{D}}
$$

Moreover

$$
t(k)=o\left(\exp -2 \beta k^{-2} \int_{0}^{b} \sqrt{V(s)} d s\right), \quad \forall \beta<1
$$

and

$$
\lim _{k \rightarrow 0} \sigma_{1}(k)=1 \quad \text { and } \quad \sigma_{n}(k)=O_{n}(k) \quad \forall n \geqq 2 .
$$

Proof. Due to the results about the W-A determinant mentioned earlier in (II.17) and the intertwining property of the eigenvalues of $r$ and $r^{D}$ shown in [CDS 1 , Chap. II], we know that $f$ is precisely the first zero of $\omega(z)$ to the right of $f^{D}(k)$. By the previous lemma we may as well look for roots of

$$
z-f^{D}=t \sigma(z) \text {. }
$$

We shall do this using Rouchés theorem and Lagrange's inversion formula (see [D, pp. 250]). So we need only to find a closed path $\gamma$ which encloses $f^{D}$ such 
that

$$
\sup \{|t \sigma(z)|, z \in \gamma\}<1 .
$$

Let $d$ be the distance of $f^{D}$ to the rest of $\sigma\left(r^{D}\right)$; we know by assumption that

$$
\lim _{k \rightarrow 0} d(k)>0 \text {. }
$$

We choose for $\gamma$

$$
\gamma=\left\{z \in \mathbb{C},\left|z-f^{D}\right|=\frac{d}{2}\right\} .
$$

The condition (1.9) is now easy to verify for sufficiently small $k$ because of (1.3) and the exponentially small estimate on $t(k)$ in Proposition II.11.

Estimates on $\sigma_{n}(k)$ are done using Cauchy's formula: for any $n \geqq 2$

$$
\sigma_{n}=\frac{(n-1) !}{2 \pi i} \int_{\gamma} \frac{\sigma^{n}(z)}{\left(z-f^{D}\right)^{n}} d z=\frac{(n-1) !}{2 \pi i} \int_{\gamma} \frac{n \sigma^{n-1}(z) \sigma^{\prime}(z)}{(n-1)\left(z-f^{D}\right)^{n-1}} d z,
$$

then

$$
\left|\sigma_{n}\right| \leqq n(n-2) ! \sup \left\{\left|\sigma^{n-1}(z)\right|\left|\sigma^{\prime}(z)\right|, z \in \gamma\right\}\left(\frac{2}{d}\right)^{n-2}=O_{n}(k),
$$

because $\sigma^{n-1}$ is uniformly bounded on $\gamma$ and

$$
\left|\sigma^{\prime}(z)\right|=\left|\sigma^{2}(z)\left(\left(\widehat{r^{D}-z}\right)^{-2} \tau, \tau\right)\right| \leqq\left|\sigma^{2}(z)\right|\left\|\left(\widehat{r^{D}-z}\right)^{-2}\right\|\|\tau\|^{2}=O(k)
$$

\section{The Degenerate Situation}

In this section we consider s.v. functions which are degenerate in the limit. Let $E_{i}$, $E_{i}^{D}, i=1,2$ be s.v. functions of $H, H^{D}$ (the labelling is defined below) such that $\lim _{k \rightarrow 0} k^{-2} E_{i}(k)=\lim _{k \rightarrow 0} k^{-2} E_{i}^{D}(k)=e^{(2)} \in \mathscr{L}$,

$$
i=1,2, \quad e^{(2)} \quad \text { being degenerate in } \mathscr{L} .
$$

As in the first section we shall work with the corresponding s.v. functions $f_{i}, \mathrm{f}_{i}{ }^{D}$ of the resolvents of the microscopic operators.

Our method applies in the two following cases

Case 1. $\forall k>0, f_{1}^{D}(k)=f_{2}^{D}(k)$.

Case 2. $\exists k_{0}>0, \quad \forall k \in\left(0, k_{0}\right) \quad t(k)<\left|f_{1}^{D}(k)-f_{2}^{D}(k)\right|$.

From now on we restrict ourself to these cases and we assume without loss of generality that

$$
\exists k_{0}>0, \quad \forall k \in\left(0, k_{0}\right), \quad f_{1}^{D}(k) \leqq f_{2}^{D}(k) .
$$

The labelling of the $f_{i}^{\mathrm{D}}$ 's as in Chap. II does correspond to the direct sum decomposition $r^{D}=r_{1}^{D} \oplus r_{2}^{D}$. The $f_{i}$ 's are labelled by their natural order.

Remark 2.1. One can easily see that

$$
\forall k>0, \quad t(k)>0,
$$


which implies in Case 2 that there is no crossing between $f_{1}^{D}$ and $\mathrm{f}_{2}^{D}$.

The proof of the result for Case 1 is similar to the one of the nondegenerate case, Theorem 1.2. So we give the corresponding theorem without proof.

Theorem 2.2. Let $E_{i}, i=1,2$, be s.v. functions of $H$ degenerate in the limit which fulfill (2.1) and $f_{i}=\left(k^{-2} E_{i}+1\right)^{-1}$. Let $E_{i}^{D}, f_{i}^{D}$ be the corresponding objects for $H^{D}$. If $f_{1}^{D}(k)=f_{2}^{D}(k)$ for any positive $k$, then one has:

$$
f_{1}(k)=f_{1}^{D}(k)=f_{2}^{D}(k), \quad \forall k>0,
$$

ii) for sufficiently small $k$

$$
f_{2}=f_{2}^{D}+\sum_{n=1}^{\infty} \frac{t^{n}}{n !} \sigma_{n}
$$

where $\sigma_{n}=\left.\frac{d^{n-1}}{d z^{n-1}}\left(\sigma(z)^{n}\right)\right|_{z=f_{1}^{D}}$.

The estimate (1.6) and (1.7) are again valid.

To investigate Case 2, we need an analog of Lemma 1.1. For its formulation we must introduce new notations:

$$
\begin{gathered}
\forall i \in\{1,2\}, \quad \tilde{\omega}_{i}=f_{i}^{D}-z-t \sigma_{i}(z), \\
\forall(i, j) \in\{(1,2),(2,1)\}, \quad \sigma_{i}(z)=\frac{z-g}{z-f_{j}^{D}} \sigma(z) .
\end{gathered}
$$

We recall that $g$ is defined in (II.29).

Lemma 2.3. There exist a neighbourhood $\mathscr{V}$ of $f^{(2)}$ and $k_{0}>0$ such that for any $k$ in $\left(0, k_{0}\right)$ and $i=1,2$, the zero sets in $\mathscr{V} \backslash\left\{f_{1}^{D}, f_{2}^{D}\right\}$ of $\omega$ and $\tilde{\omega}_{i}$ coincide.

Proof. One has obviously

$$
\omega(z)=\left(f_{i}^{D}-z\right)^{-1} \sigma^{-1}(z) \tilde{\omega}_{i}(z) \quad i=1,2 .
$$

The rest of the proof is similar to that of Lemma 1.1.

We are ready for the following

Theorem 2.4. Let $E_{i}, i=1,2$, be s.v. functions of $H$ degenerate in the limit which fulfill (2.1), and $f_{i}=\left(k^{-2} E_{i}+1\right)^{-1}$. Let $E_{i}^{D}, f_{i}^{D}$, be the corresponding objects for $H^{D}$. In addition assume that

$$
\exists k_{0}>0, \quad \forall k \in\left(0, k_{0}\right), \quad t(k)<f_{2}^{D}-f_{1}^{D},
$$

where $t=t_{1}+t_{2}$ is the total tunneling parameter. Then for sufficiently small $k$ one has

$$
f_{i}=f_{i}^{D}+\sum_{n=1}^{\infty} \frac{t^{n}}{n !} \sigma_{i, n}, \quad i=1,2
$$

where

$$
\sigma_{i, n}=\left.\frac{d^{n-1}}{d z^{n-1}}\left(\sigma_{i}(z)\right)^{n}\right|_{z=f_{i}^{D}}, \quad i=1,2
$$


Moreover

$$
t_{i}(k)=o\left(\exp -2 \beta k^{-2}\left|\int_{x_{i}}^{b} V(s)^{1 / 2} d s\right|\right) \quad \forall \beta<1
$$

and

$$
\sigma_{i, n}=O_{n}\left(\left(f_{2}^{D}-f_{1}^{D}\right)^{1-n}\right) \quad n \geqq 1 .
$$

Proof. By the same remark as the one in the beginning of the proof of Theorem 1.2 we know that $f_{i}$ is precisely the first zero of $\omega$ to the right of $f_{i}^{D}, i=1,2$. By Lemma 2.3 we may as well seek this zero as a root of

$$
z-f_{i}^{D}=t \sigma_{i}(z), \quad i=1 \quad \text { or } 2 .
$$

To recover $f_{i}$ we shall use again Rouché's theorem and Lagrange's inversion formula (see [D, pp. 250]). ${ }^{3}$ We must find a closed path $\gamma_{i}$ which encloses $f_{i}^{D}$ such that

$$
t<\inf \left\{\left|\frac{z-f_{i}^{D}}{\sigma_{i}(z)}\right|, z \in \gamma_{i}\right\} .
$$

But it is sufficient to fulfill

$$
t<\frac{\inf \left\{\frac{\left(z-f_{1}^{D}\right)\left(z-f_{2}^{D}\right)}{z-g}, z \in \gamma_{i}\right\}}{\sigma^{+}\left(\gamma_{i}\right)}
$$

where $\sigma^{+}\left(\gamma_{i}\right)=\sup \left\{|\sigma(z)|, z \in \gamma_{i}\right\}$. Because of the assumption (2.8) it is sufficient to show that there exist $\gamma_{i}$ 's such that

$$
\lim _{k \rightarrow 0} \sigma^{+}\left(\gamma_{i}\right)=1
$$

and

$$
\inf \left\{\frac{\left(z-f_{1}^{D}\right)\left(z-f_{2}^{D}\right)}{z-g}, z \in \gamma_{i}\right\}=f_{2}^{D}-f_{1}^{D} .
$$

There is no difficulty to find suitable $\gamma_{i}$ 's. We show an example in the appendix.

The estimates on $t_{i}(k)$ are shown in Proposition II.11, whilst those on $\sigma_{i, n}$ are done using Cauchy's formula:

$$
\begin{aligned}
\left|\left(\frac{d^{n-1}}{d z^{n-1}} \sigma_{i}^{n}\right)\left(f_{i}^{D}\right)\right| & =\left|\frac{(n-1) !}{2 \pi} \int_{\gamma_{i}} \sigma^{n}(z) \frac{(z-g)^{n}}{\left(z-f_{1}^{D}\right)^{n}\left(z-f_{2}^{D}\right)^{n}} d z\right| \\
& \leqq \frac{(n-1) !}{2 \pi}\left|\gamma_{i}\right| \sup \left\{\left|\sigma^{n}(z)\right|, z \in \gamma_{i}\right\}\left(f_{2}^{D}-f_{1}^{D}\right)^{-n} \quad \text { due to (2.17) } \\
& =O_{n}\left(\left(f_{2}^{D}-f_{1}^{D}\right)^{-n+1}\right) .
\end{aligned}
$$

because the length $\left|\gamma_{i}\right|$ of $\gamma_{i}$ is $O\left(\left(f_{2}^{D}-f_{1}^{D}\right)\right)$ [see Appendix (A.2)].

3 In fact we use an obvious generalization of what Dieudonné writes in his book: we simply take a connected domain instead of a disk 
Remark 2.5. To unify the presentation of Theorem 2 we have chosen to expand the shifts $f_{i}-f_{i}^{D}$ in power of the total tunneling parameter. Nevertheless one should not believe that the leading term of $f_{i}-f_{i}^{D}$ is $t$ because as

This gives

$$
\sigma_{i, 1}=\alpha_{i}^{2} \sigma\left(f_{i}^{D}\right) \text {. }
$$

$$
f_{i}-f_{i}^{D}=t_{i} \sigma\left(f_{i}^{D}\right)+\ldots
$$

\section{Concluding Remarks}

\section{A More Precise Estimate on the Tunneling Parameter: A Conjecture}

In the case of symmetric double-wells we can say more on the tunneling parameters. If we choose $b=\frac{1}{2}\left(x_{1}+x_{2}\right)$, then we have $t_{1}(k)=t_{2}(k)=\frac{1}{2} t(k)$. If $e_{i}$, $i=1,2$, denote two s.v. functions of $h(k)$ such that $\lim _{k \rightarrow 0} e_{1}(k)=\lim _{k \rightarrow 0} e_{2}(k)$, then using results of Theorem III.2.2 we obtain

$$
\lim _{k \rightarrow 0} \frac{\left|e_{1}(k)-e_{2}(k)\right|}{t(k)}=1 .
$$

From the behaviour of $\left|e_{1}(k)-e_{2}(k)\right|$ proved in [H1] and announced in [S2] we deduce that

$$
\lim _{k \rightarrow 0} k^{2} \log t(k)=-\int_{x_{1}}^{x_{2}} V(s)^{1 / 2} d s .
$$

This allows us to make the following conjecture.

Conjecture. The tunneling parameters defined in Definitions II.6 and II.8 satisfy:

$$
\lim _{k \rightarrow 0} k^{2} \log t_{i}(k)=-2\left|\int_{x_{i}}^{b} V(s)^{1 / 2} d s\right|, \quad i=1,2,
$$

where the nondegenerate case is described by $i=1$.

\section{Multi-Instanton Expansion}

In the case of symmetric double-wells we want to mention that the expansion (III.2.4) of $\left|f_{2}-f_{1}\right|$ has at least formally the structure of a multi-instanton expansion. This last expression is borrowed from Zinn-Justin [Z]. We can read in [Z] that the leading term of $\left|e_{2}-e_{1}\right|$ is the "one instanton contribution." Therefore because of (1.1) we can say that the tunneling parameter $t(k)$ corresponds to the one instanton contribution. Still following [Z] we see that $t(k)^{n}$ corresponds to the $n$-instanton contribution. Obviously an expansion of the same type is valid for $\left|e_{2}-e_{1}\right|$.

\section{Possible Extensions}

To simplify the matter we have chosen to show our method to compute tunneling effects in the frame of the harmonic approximation. In fact there are no extra 
difficulties to consider flatter minima of the potential $V$. Also the method can be applied easily to operators $H(k)$ with more general types of boundary conditions on $\partial \Omega$ than Dirichlet's one.

Among the extensions on which our method does not apply directly we mention tunneling between more than two wells and tunneling in $n$ dimensions. The former has been considered in [H2] and [JMS] in some special cases. They give only the leading asymptotics to the shift induced by tunneling. Some results about the latter are announced in [S2].

\section{Appendix}

\section{Bound on Boosted Green's Functions}

Theorem A.1.1. Let $\Omega$ be an open interval of $\mathbb{R}$ and $V \in L_{\mathrm{loc}}^{1}(\Omega), V$ nonnegative. Let $H$ be the positive self-adjoint operator with symbol $-\Delta+V$ obtained by Friedrichs extension. Let $U(\alpha)$ be the multiplication operator by $\exp i \alpha \int_{x_{0}}^{x} V(s)^{1 / 2} d s$ for $x_{0}$ in $\Omega$ and $|\operatorname{Im} \alpha|<1$. Let $g(\alpha ; x, y)$ be the Green's function of $H(\alpha)=U(\alpha) H U(\alpha)^{-1}$ at $z=-1$, then

$$
\forall(x, y) \in \Omega^{2},\|g(\alpha ; \cdot, y)\| \text { and }\|g(\alpha ; x, \cdot)\| \leqq C_{\Omega}^{1 / 2} \leqq 1,
$$

where $C_{\Omega}=\frac{1}{2}\left(|\Omega|^{-2}+4\right)^{1 / 2}-\frac{1}{2}|\Omega|^{-1}$ and $|\Omega|$ denotes the measure of $\Omega$.

Proof. $H(\alpha)$ is the self-adjoint holomorphic family of type $B$ (see [Ka, p. 393]) associated to the quadratic form

$$
t(\alpha)[u]=\|\nabla u\|^{2}+\left(1+\alpha^{2}\right)\left\|V^{1 / 2} u\right\|^{2}+\alpha\left[\left(i \nabla u, V^{1 / 2} u\right)+\left(V^{1 / 2} u, i \nabla u\right)\right],
$$

with the form domain of $H$. This was shown in [CDS1, Lemma 3.5 of Chap. III]. Now if $R(\alpha)$ denotes the resolvent $(H(\alpha)+1)^{-1}$, one has because $H(\alpha)$ is $m$-sectorial with vertex zero:

$$
\|R(\alpha)\| \leqq 1, \quad \forall|\operatorname{Im} \alpha|<1 .
$$

As $U(\alpha)$ is unitary for real $\alpha$ it is sufficient to make the proof for $\alpha \in i \mathbb{R}$. Let $C$ be an arbitrary positive constant. Then by the Sobolev inequality one has

$$
|(g(\alpha ; x, \cdot), u)|^{2}=|(R(\alpha) \bar{u})(x)|^{2} \leqq\left(C+|\Omega|^{-1}\right)\|R(\alpha) \bar{u}\|^{2}+C^{-1}\left\|(R(\alpha) \bar{u})^{\prime}\right\|^{2} .
$$

Now we choose $C=C_{\Omega}$ such that $C+|\Omega|^{-1}=C^{-1}$. Thus

$$
\begin{aligned}
|(g(\alpha ; x, \cdot), u)|^{2} & \leqq C_{\Omega}(1+\operatorname{Re} t(\alpha))[R(\alpha) \bar{u}] \\
& \leqq C_{\Omega}|((H(\alpha)+1) R(\alpha) \bar{u}, R(\alpha) \bar{u})| \\
& \leqq C_{\Omega}|(\bar{u}, R(\alpha) \bar{u})| \leqq C_{\Omega}\|R(\alpha)\|\|u\|^{2} .
\end{aligned}
$$

So $\|g(\alpha ; x, \cdot)\|^{2} \leqq C_{\Omega}$. That $C_{\Omega}$ is bounded by one uniformly in $|\Omega|$ is obvious.

\section{Suitable Contours $\gamma_{i}$ for the Proof of Theorem 2.4, Chap. III}

The choice of $\gamma_{i}$ 's which fulfill (III.2.17) is obviously not unique. To explain our choice let us introduce some convenient notations:

$$
Z=z-g, \quad T(Z)=Z-B^{2} Z^{-1}, \quad B=\alpha_{1} \alpha_{2} \Delta, \quad \Delta=\left|f_{2}^{D}-f_{1}^{D}\right|,
$$


with which (III.2.17) becomes

$$
\inf \left\{|T(Z)-B|, Z \in \gamma_{i}-g\right\}=\Delta .
$$

Because of the two critical points of $T(Z)$, namely $Z= \pm i B$, one can only expect that

$$
\inf \left\{|T(Z)-B|, Z \in \gamma_{i}-g\right\} \leqq \Delta .
$$

The transformation $Z \rightarrow T(Z)$ is so simple that we give the $\gamma_{i}$ 's by the following pictures with some further comments afterwards:
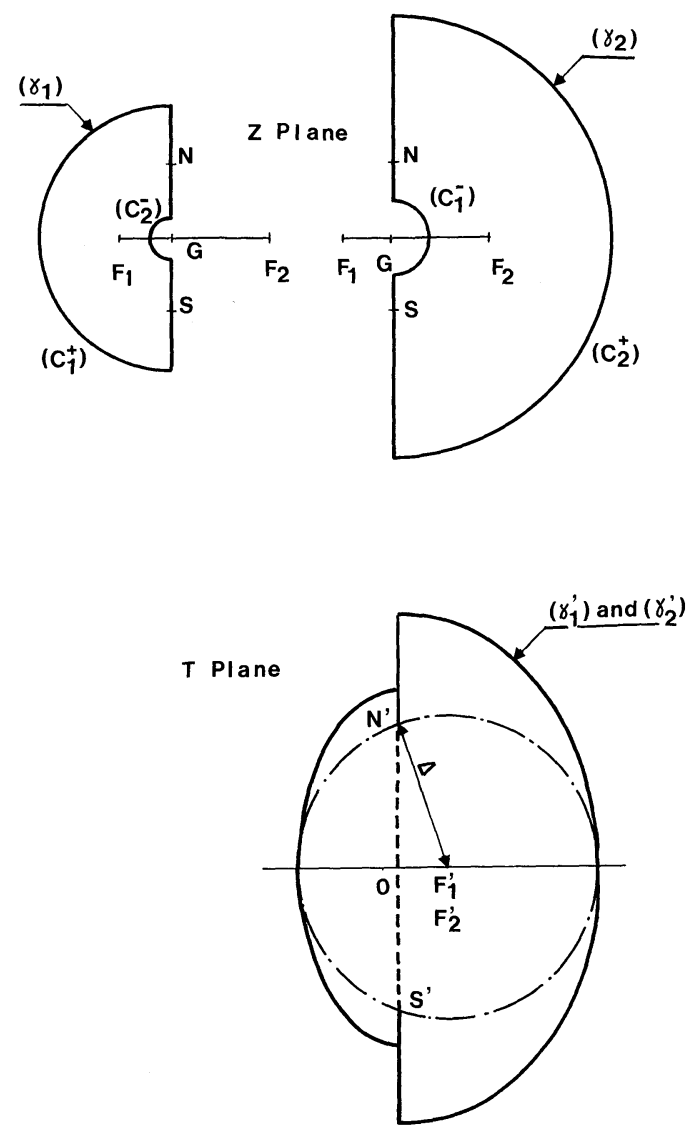

Fig. 2. The drawing is made for $\alpha_{1}^{2}=1 / 3, \alpha_{2}^{2}=2 / 3$

$F_{1}, F_{2}, G, N, S$, are, respectively, the points corresponding to $f_{1}^{D}, f_{2}^{D}, g, g+i B$, $g-i B$ in the $z$ plane whilst their image under $Z \rightarrow T(Z): F_{1}^{\prime}, F_{2}^{\prime}, G^{\prime}, N^{\prime}, S^{\prime}$ are the points corresponding to $0,0, \infty, 2 i B,-2 i B$ in the $T$ plane. $\left(C_{1}^{ \pm}\right)$and $\left(C_{2}^{ \pm}\right)$are, respectively, the circles with center $G$ and radius

$$
r_{1}^{ \pm}=\left( \pm \alpha_{1}^{2}+\alpha_{1}\right) \Delta, \quad r_{2}^{ \pm}=\left( \pm \alpha_{2}^{2}+\alpha_{2}\right) \Delta
$$


The domains enclosed by $\gamma_{1}$ and $\gamma_{2}$ are the smallest ones of that shape whose images contain the ball centered at zero with radius $\Delta$. We have chosen that shape because circles centered at the origin in the $Z$ plane are transformed into simple ellipses. We have checked (III.2.17). To verify (III.2.16) one must notice that $r_{1}^{ \pm}$and $r_{2}^{ \pm}$go to zero with $k$; therefore this follows from the uniform convergence of $\sigma$ on a small neighbourhood of $f^{(2)}$ already shown in Lemma III.1.1.

Acknowledgements. Pierre Duclos acknowledges the hospitality of the Free University of Berlin and the DFG for Financial Support.

\section{References}

[AG] Akhiezer, N.I., Glazman, I.M.: Theory of linear operators in Hilbert space. New York: Frederick Ungar Publishing Co. Inc. 1961

[CDS0] Combes, J.M., Duclos, P., Seiler, R.: The Born Oppenheimer approximation, pp. 185-212 of Rigorous Atomic and Molecular Physics, Proceedings (1980). Wightman, A.S., Velo, G. (eds.). New York: Plenum Press 1981

[CDS1] Combes, J.M., Duclos, P., Seiler, R.: Krein's formula and one dimensional multiple well. J. Funct. Anal. 52, 257-301 (1983)

[D] Dieudonné, J.: Calcul infinitésimal, Chap. VIII.7. Paris: Herman 1968

[Da] Davies, E.B.: Double well hamiltonians. Preprint 1983

[H1] Harrell, E.M.: Double wells. Commun. Math. Phys. 75, 239-261 (1980)

[H2] Harrell, E.M.: The band structure of a one-dimensional, periodic system in a scaling limit. Ann. Phys. (NY) 119, 351-369 (1979)

[JMS] Jona-Lasinio, G., Martinelli, F., Scoppola, E.: New approach to the semiclassical limit of quantum mechanics. I. Multiple tunnelings in one dimension. Commun. Math. Phys. 80, 223-254 (1981)

[Ka] Kato, T.: Perturbation theory for linear operators. Berlin, Heidelberg, New York: Springer 1966

[Kr] Krein, M.: Über Resolventen hermitescher Operatoren mit Defektindex $(m, m)$. Dokl. Akad. Nauk SSSR 52, 657-660 (1946)

[P] Polyakov, A.N.: Quark confinement and topology of gauge theories. Nucl. Phys. B 120, 429-458 (1977)

[R-R] Richard, J.L., Rouet, A.: Complex saddle points versus dilute-gas in the double well anharmonic oscillator. Nucl. Phys. B 185, 47-60 (1981)

[S1] Simon, B.: Semiclassical analysis of low lying eigenvalues. I. Nondegenerate minima: asymptotic expansions. Ann. Inst. Henri Poincaré 38, 295-307 (1983)

[S2] Simon, B.: Instantons, double wells, and large deviations. Bull. Am. Math. Soc. 8, 323-326 (1983)

[W] Witten, E.: Supersymmetry and Morse theory. J. Differential Geometry 17, 661 (1982)

[Z] Zinn-Justin, J.: Multi-instanton contributions in quantum mechanics. Nucl. Phys. B 192, 125-140 (1981); and, The principles of instanton calculus: a few applications, Les Houches Lecture Notes 1982, Preprint DPH-T/80-82 (Oct. 1982)

Communicated by B. Simon

Received June 14, 1983; in revised form July 14, 1983 
